\title{
Preface of the Special Issue "Anti-Tumor CAR-T Cell Therapy"
}

Immunotherapeutic strategies have benefited cancer treatment of the hematological system and even solid tumors in recent years. The immunological approach includes immune checkpoint inhibitors, cancer vaccines, adoptive cell therapy, cytokines, and monoclonal antibodies. Also, chimeric antigen receptor (CAR)-T cell therapy is even more promising. This type of treatment involves the genetic manipulation of the patient's $\mathrm{T}$ cells to express a specific TCR receptor that recognizes the tumor antigen(s) and thus triggers a precise and effective cytotoxic antitumor response.

This Special Issue focuses on the processes for the generation of CAR-T cells, definition/identification of specific tumor antigens, genetic engineering of $\mathrm{T}$ cells, and their clinical application in oncology.

Review articles cover aspects of the recent progress in cancer immunotherapy, highlighting the use of genetically engineered $T$ cells to target selected tumor antigens, the reduction of side effects, and increasing the effectiveness of oncologic treatment. They also discuss the bottleneck of this approach, which is the identification the specific tumor antigen(s).

The seven contributions from researchers from different countries are outlined below.

Chen and Jiang's article, "Current Challenges and Strategies for Chimeric Antigen Receptor-T-Cell Therapy for Solid Tumors," discusses the use of CAR-T cells against solid tumors focusing on CAR-T cell infiltration and survival, and difficulties faced, such as vasculature and stromal barriers, hypoxia, and high metabolism rate found in solid tumors, as well as immunosuppression.

Pasca et al.'s article, "Implications of TET2 in CAR-T Cell Activity and Target Response to CAR-T Cell Therapy: Lessons Learned from $\mathrm{T}$ Cells," discusses the effects that TET2 can have on $\mathrm{T}$ cells, macrophages interacting with $\mathrm{T}$ cells, and nonimmune cells. Among other considerations, the authors discuss the influence of TET2 activity on macrophage polarization, which at the tumor site might influence CAR-T cell activity.

In "Current Headway in Cancer Immunotherapy Emphasizing the Practice of Genetically Engineered T Cells to Target Selected Tumor Antigens," Ray and Mukherjee discuss neoantigens' expression by tumor cells, which might be immunogenic. However, the tumor antigens can be either target for immunotherapeutic strategy through CAR-T cells or act as immunosuppressors and be a cause of tumor escape. The authors also discuss the challenges of CAR-T cell therapy regarding its specificity and effectiveness.

Barros's article, "In Search for an Ideal CAR-T Cell Antigen Target," discusses the challenge imposed by the heterogeneity of the expression of neoantigens by solid tumors on CAR-T cell therapy. The author also states that many antigens are shared between solid tumors and healthy cells, which may cause off-target effects from CAR-T cells. The article also considers that a combination of antigens could represent an alternative to overcome these limitations.

In "CAR-T Cell Therapy: Challenges and Optimization," Luo et al. discuss the safety and efficacy of the current CAR-T cell therapy and strategies to maximize the antitumor effectiveness while minimizing the adverse reactions.

Lima et al.'s article, "Strategies to Enhance the Therapeutic Efficacy, Applicability, and Safety of Genetically Engineered Immune Cells," discusses the obstacles that still restrict the antitumor efficacy and impair the general use of CAR-T cells. They discuss challenges including the limited activity of CAR-T cells in vivo, antigen escape, and therapy-related toxicity. Nevertheless, the authors also provide perspectives to overcome these limitations and increase the use of genetically engineered $\mathrm{T}$ cells to treat cancer.

Finally, Abdo et al.'s article, "Structural Determinants of Chimeric Antigen Receptor Design," critically discusses modifications in the chimeric antigen receptor (CAR) that were made to improve its 
clinical benefits, for example, alterations in each of the CAR molecule domains, aiming for modulation of the binding affinity or the use of costimulatory molecules' ligands and control of the intracellular signaling.

This Special Issue offers a critical reading of the present CAR-T cell research given the current knowledge of the tumor cells' biology and genetics, molecular biology, and immunology directed toward cancer treatment.

Guest Editor:

Geraldo Aleixo Passos

University of São Paulo, Ribeirão Preto Campus, SP, Brazil 